\title{
CATASTROPHIC DISRUPTION OF SOLID BODIES BY COLLISION - EXPERIMENTAL APPROACH -
}

\author{
A. FUJIWARA \\ Department of Physics, \\ Kyoto University, \\ Sakyo-ku, Kyoto 606, Japan
}

\begin{abstract}
The results on mass, velocity, shape, and rotation of the fragments produced in the disruption experiments by impact are surveyed. Some future works are also suggested.
\end{abstract}

\section{Introduction}

Study of the outcomes of collisions between the solid bodies is crucial to understand the nature, origin, and evolution of interplanetary dust particles in many respects: (1) Some of the dust particles are undoubtedly supplied as the outcome of the asteroid-asteroid, or asteroid-dust collisions as found as IRAS dust bands, which are closely connected with some of asteroid families, and the structure and evolution of the dust bands are controled by the collisional processes (Sykes et al., 1989). (2) Collisions among dust particles are believed to play an important role to supply the $\beta$-meteoroids, which are observed in the recent direct detection of dust particles by spacecrafts. (3) Dust particles are considered to be the end products of the collisional processes among the solid components of various size classes since the formation of the solar system.

To solve these collisional evolution and identify the sources of the interplanetary dust particles, the most fundamental quantities to be investigated on the collisional outcomes are: (1) mass (size) distribution of the fragments, and (2) velocity distribution of the fragments. Other quantities are also useful for some purposes; for examples, informations of the fragment shape is useful to identify whether dust particles are collisional origin or not, by the recovered samples or scattering properties of the dust particles, and to evaluate the Yarkovski effect working on the spinning dust particles, rotational frequencies of the collisional fragments must be known. Impact phenomena are classified into two classses: cratering and disruption. Concerning the size and velocity distribution of the fragments in cratering, Gault and Heitowit's (1963) classical work is still useful and valuable, although more systematic works are strongly desired. On the contrary, studies concerning the impact disruption have made great progress in the past decade. In this review, we mainly focus on the outcomes of the impact destruction found through experiments. Main

\section{1}

A.C. Levasseur-Regourd and H. Hasegawa (eds.), Origin and Evolution of Interplanetary Dust, 361-366.

(c) 1991 Kluwer Academic Publishers, Printed in Japan. 
experimental results and scaling laws in the context of the catastrophic collision of asteroididal bodies until 1989 are summarized in Fujiwara et al.(1989). In this paper, some of the important points and recent topics are reviewed.

\section{Experimental Methods}

Most commonly made experiments are laboratory impact experiments using powder guns and sigle-stage or two-stage light-gas guns which can launch typically cm-sized projectiles up to several $\mathrm{km} / \mathrm{s}$. Shaped charge method is also used in higher velocity experiments (about $9 \mathrm{~km} / \mathrm{s}$ )(Capaccioni et al.,1986). Materials used for the projectiles and the targets are metals, rocks, and ices. Recently disruption experiments in the open air using explosives have been conducted by an Italian group (Martelli et al., 1990). In these experiments aerodynamical drag for small fragments should be taken into account, while the experiments using guns are carried out in the vacuum state. For the dust particles, electrostatic dust accelerators are used, but the studies for the disruption are quite limited (McDonnell, 1976).

\section{Fragment size}

\subsection{ENERGY FOR DISRUPTION AND THE LARGEST FRAGMENTS}

Impact energy required to disrupt the targets consisting of various kind of materials and the degree of fragmentation were investigated by many authors (See Fujiwara et al., 1989). Most of rocks start to disrupt at E/M-value (E; impact energy, M; target mass) of about several times $10^{6} \mathrm{erg} / \mathrm{g}$, while ices disrupt at one order of magnitude smaller value. For metals (e.g. iron) disruption occurs at around $10^{8}$ $\mathrm{erg} / \mathrm{g}$. The mass fraction of the largest fragment to the target mass is roughly inversely proportional to the $\mathrm{E} / \mathrm{M}$-value for various materials.

\subsection{SIZE DISTRIBUTION OF THE FRAGMENTS}

It has been traditionally assumed in many papers that the size distribution is expressed by a power law;

$$
n(m) d m=C m^{-\alpha} d m
$$

where $n(m) d m$ is the number of fragments of mass in the range of $m$ to $m+d m$. The index has been conventionally assumed to be 0.8 in many references. However, the detailed studies show that is a function of fragment size and impact energy or more appropriate scaling parameters. Takagi et al.(1984) showed that the size spectrum consists of three segments and the power indices for these segments are expressed as a function of a scaling parameter $\mathrm{P}_{I}$ which is proposed as a good scaling parameter by Mizutani et al.(1990) and expressed as $\mathrm{P}_{I}=\mathrm{P}_{0} \mathrm{~V}_{0 p} / \mathrm{YV}_{0 t}\left(\mathrm{~V}_{0 p}\right.$; initial volume of a projectile, $\mathrm{V}_{0 t}$; initial volume of a target, $\mathrm{Y}$; yield strength of the target). The results show that the exponent changes with the parameter $\mathrm{P}_{I}$. In the regime III (the small size region) most of data are slightly less than 0.8 , while in the regime I (region of the largest fragment size), they take very large values and increase with the parameter $\mathrm{P}_{I}$. 


\section{Velocity Distribution}

Many investigators consider that velocity distribution of the fragments (relation between mass and velocity of individual fragments) is the most fundamental quantity to understand the collisional evolution of the solid particles in the solar system. However, the distribution in the whole size range has not yet been understood because of the experimental difficulties. Recently Nakamura and Fujiwara (1990)

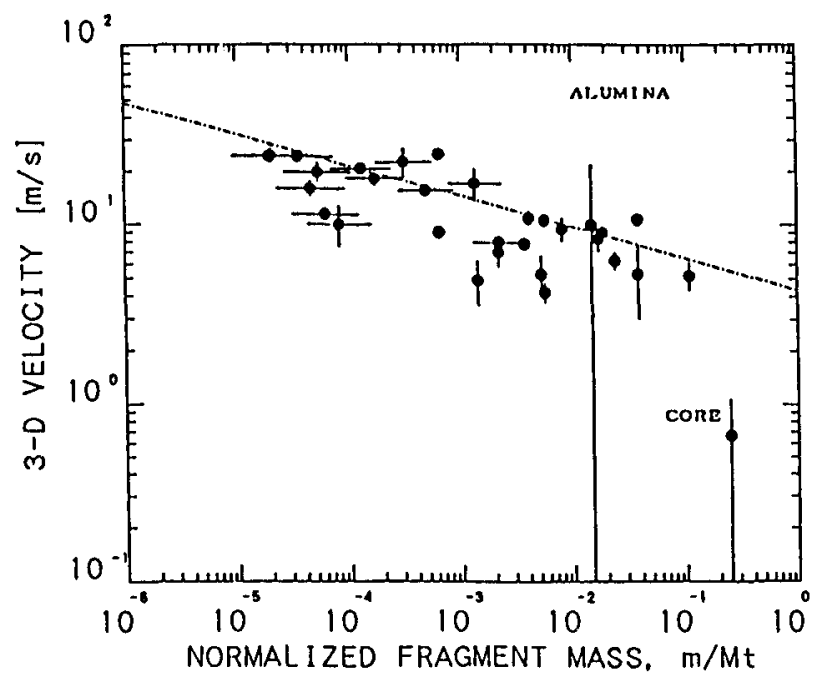

Figure 1. Velocity distribution of the alumina fragments. Velocity and mass of the projectile are $3.8 \mathrm{~km} / \mathrm{s}$ and $0.7 \mathrm{~g}$, respectively. Target diameter is $6 \mathrm{~cm}$. Nakamura and Fujiwara(1990).

took high-speed movie pictures of the fragmentation of an alumina ball and a basalt ball impacted by the nylon projectiles of $0.7 \mathrm{~cm}$ in diameter in the vacuum space. The $2-\mathrm{D}$ velocity and $3-\mathrm{D}$ velocities were determined for the fragment size down to a few $\mathrm{mm}$. The example of the $3-\mathrm{D}$ velocity distribution for the alumina target is shown in Fig.1. The best-fitted line is expressed by $-1 / 6$ th power of the fragment mass. These results are not inconsistent of the results by Davis and Ryan(1989). It is still not clear how this velocity distribution depends on the various impact parameters (masses and material properties of both the target and the projectile, and collisional velocity). However, the data on the velocity of the antipodal points of the target were obtained as a representative velocity (Fujiwara and Tsukamoto, 1980; Takagi et al.,1984), where the antipodal point is the opposite point on the target of the impact point. The data are shown in Fig.2. The velocity depends on the 0.76 th power of $\mathrm{E} / \mathrm{M}$, and it is found from the figure that the data are well scaled by the Mizutani scaling parameter. 

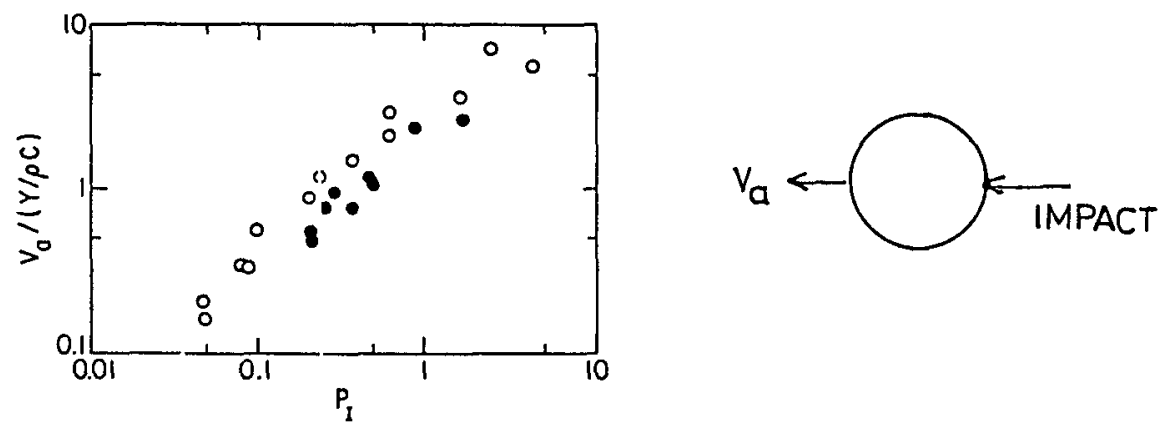

Figure 2. Velocity of fragments from the antipodal point $\mathrm{V}_{a}$. $\mathrm{PI}$ is Mizutani parameter. $\mathrm{C}$ and $\rho$ are sound velocity and density of the data $(2.7 \mathrm{~km} / \mathrm{s})$. Filled circle; the Takagi and Mizutani data $(<1 \mathrm{~km} / \mathrm{s})$. From Fujiwara et al.(1989).

\section{Shape of the Fragments}

Shape of the fragments is especially of interest for the study of dust particles, because it affects the scattering properties of the dust particles and it also becomes a clue to assign the origin of the dust particles. Many laboratory experiments show shapes of the fragments distributing around a very peculiar average shape(Fig. 3 ); the average ratio for the longest, intermediate, and short axis is $2: 2^{1 / 2}: 1$. Hence,
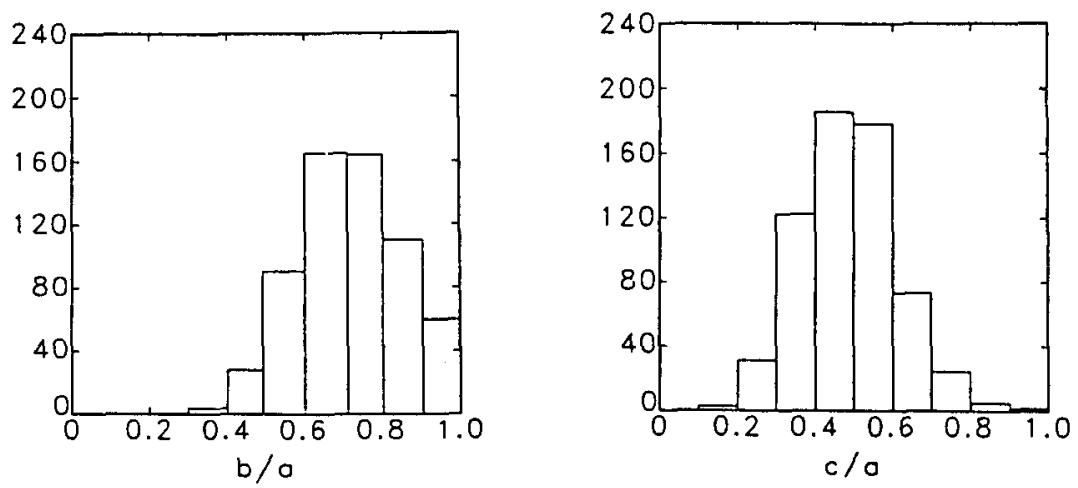

Figure 3. Histogram for b/a and c/a. Fujiwara et al.(1989).

if the dust particles are originated from collisional processes, the most probable shape of the dust particles will take this average value. There are two experiments which violate this very stable rule; one is Lange and Ahrens (1981) for ice targets, and another is the recent large scale experiments made by using explosives in the open air by Martelli et al. (1990). The reason of the deviation from the standard 
shape rule in these experiments is not clear, but the possibility that the original samples may have some anisotropy is not excluded.

\section{Rotation of the Fragments}

Rotational properties affect on the dust particles through Yarkovski effect. The data on the rotational frequency of the fragmented bodies are still limited to the one obtained by Fujiwara and Tsukamoto(1981). It is noted the fragments rotate at very high frequencies; for example, the fragments of several $\mathrm{mm}$ size rotate as high as a few hundreds rps. The maximum frequency rate is almost one order-ofmagnitude less than the frequency for the rotational burst.

\section{Future Works}

There are many works to be done in the future on the impact phenomena connected with the interplanetary dust particles.

(1) Data of the impact experiments for wide varieties of physical parameters; varying density, size, strength of both targets and projectiles are required.

(2) Kinematical data of the fragments (velocity and spin distribution of the fragments, energy partition rate into various energy modes, angular momentum and momentum transfer rate) are still deficient.

(3) Impact experiments using various kind of icy and organic bodies are required.

(4) Collision between core-mantle bodies is of interest, because interstellar dust particles are considered to have such structure and also cometary dust particles may have similar structures.

(5) Most of experiments are aimed at the applications to the collisional evolution of asteroidal bodies. Semi-empirical laws or scaling laws proposed( Paolicchi et al., 1989; Housen and Holsapple, 1990; Mizutani et al., 1990) are intended to know how the laboratory scale experiments $(10 \mathrm{~cm}$ order bodies) can be extended to the $10^{6-7} \mathrm{~cm}$-sized bodies. From the viewpoint of the interest in the dust particles, it is necessary to extend the scaling law to an order of $10^{-4} \mathrm{~cm}$ bodies. Especially interesting is to know how the disruption phenomena change for the object whose size is less than the fundamental size of the grains which constitute the body. McDonnell(1976) impacted $10^{-11} \mathrm{~g}$ particles (velocity $4 \mathrm{~km} / \mathrm{s}-15 \mathrm{~km} / \mathrm{s}$ ) on a previously raised chip-essentially free standing- of a polished lunar sample. He suggested the catastrophic rupture limit of the body size is $<4$ times the primary crater diameter. More experiments are required in this size range.

(6) Some of the Brownlee particles are aggregates of less smaller particles. Therefore collisions for these aggregates or rubble piles should be of great importance. Some experiments using rubble pile targets were made in the slow velocity range (Hartmann, 1980), and the results suggest the outcomes are strongly dependent on the initial size distribution of the rubbles. Moreover, recently, fractal structure is also proposed for the grain. To determine the cross sections for accretion or destruction using fractal grains are necessary in the discussion of growth rate of the grain in the space. 


\section{Acknowledgment}

The author wishes to thank Dr. McDonnell for his valuable comments.

\section{References}

Capaccioni, F., P. Cerroni, M. Coradini, M. DiMartino, P. Farinella, E. Flamini, G. Martelli, P. Paolicchi, P. N. Smith, A. Woodward, and V. Zappala (1986) 'Asteroidal Catastrophic Collisions Simulated by Hypervelocity Impact Experiments' Icarus 66, 487-514.

Davis, D. and E. Ryan (1989) 'On Collisional Disruption: Experimental Results and Scaling Laws', Icarus 83, 156-182.

Fujiwara, A. and A. Tsukamoto (1981) 'Rotation of Fragments in Catastrophic Impact', Icarus 48, 329-334.

Fujiwara, A., P. Cerroni, D. R. Davis, E. Ryan, M. DiMartino, K. Holsapple, and K. Housen (1989) 'Experiments and Scaling Laws on Catastrophic Collisions' in R. P. Binzel, T. Gehrels, and M. S. Matthews (eds.), Asteroids II, Univ. Arizona Press, Tucson, pp. 240-265.

Gault, D. E. and E. D. Heitowit (1963) 'The Partition of Energy for Hypervelocity Impact Craters Formed in Rock', Proc. 6th Hypervelocity Impact Symp., 2 (Cleveland, OH, Firestone Rubber Co.), pp. 419-456.

Hartmann, W. K. (1980) 'Continued Low-velocity Impact Experiments at Ames Vertical Gun Facility: Miscellaneous Results' Lunar and Planet. Sci. Conf. XI Abstract 404-406.

Housen, K. and K. Holsapple (1990) 'On the Fragmentation of Asteroids and Planetary Satellites', Icarus $84,226-253$.

Lange, A., and T. J. Ahrens (1981) 'Fragmentation of Ice by Low-velocity Impact', Proc. Lunar Planet. Sci. Conf. 12, 1667-1687.

Martelli, J., P. Rothwell, P. N. Smith, I. Giblin, J. Martinsson, E. Ducrocq, M. Wettstein, M. DiMartino, P. Farinella (1990) 'Jets of Fragments from Catastrophic Break-up and Their Astrophysical Implications' presented in this colloquium.

McDonnell, J. A. M. (1976) 'Finite 'Target Hypervelocity Impact Measurements at Microscale Dimensions: Implications for Regolith Impacts', in Papers Presented to the Symposium on Cratering Mechanics. The Lunar Science Institute, Houston, pp. 73-75.

Mizutani, H., Y. Takagi, and S. Kawakami (1990) 'New Scaling Law on Impact Fragmentation', Icarus 87, 307-326.

Nakamura, A. and A. Fujiwara (1990) 'Velocity Distribution of Fragments Formed in Simulated Collisional Disruption' submitted to Icarus.

Paolicchi, P., A. Celino, P. Farinella, and V. Zappala (1989) 'A Semiempirical Model of Catastrophic Breakup Processes', Icarus 77, 187-212.

Sykes, M. V., R. Greenberg, S. F. Dermott, P. D. Nicholson, J. A. Burns, and T. N. Gautier, III (1989) 'Dust Bands in the Asteroid Belt', in R. Binzel, T. Gehrels, and M. Shapley (eds.) Asteroids II, Univ. of Arizona Press, Tucson, 336-367.

Takagi, Y., Mizutani, H., and S. Kawakami (1984) 'Impact Fragmentation Experiments of Basalts and Pyrophyllites', Icarus 59, 462-477. 